\title{
Matarratón (Gliricidia sepium), Botón de oro (Tithonia diversifolia) y Morera (Morus alba) tres especies forrajeras usadas como alternativa en la alimentación de conejos: revisión sistémica y metanálisis.
}

\author{
Noitier Enrique Cano Cardozoํ, Francis Liliana Valencia Trujillo² \\ ${ }^{1}$ Esp. Nutrición Animal Sostenible, Universidad Nacional Abierta y Distancia - UNAD. ${ }^{2}$. Docente Escuela de Ciencias \\ Agrícolas, Pecuarias y del Medio Ambiente, Universidad Nacional Abierta y a Distancia - UNAD. \\ 1noircano@hotmail.com, 2francis.valencia@unad.edu.co
}

\section{Introducción}

En Colombia, se cuenta con una gran variedad de fuentes alimenticias con alto valor nutricional que no han sido aprovechadas adecuadamente, de las cuales podemos resaltar el Matarraton (Gliricidia sepium), botón de oro (Tithonia diversifolia) y morera (Morus alba). La alta disponibilidad de plantas probadas 0 potencialmente útiles para conejos, sustenta la posibilidad de incluirlas en mezclas dietéticas balanceadas preparadas en la granja, para aprovechar la capacidad herbívora de esta especie animal. El costo de los alimentos concentrados comerciales ha generado la búsqueda de estrategias basadas en el uso de materias primas no convencionales, que permitan obtener una mayor rentabilidad en la cunicultura.

Este trabajo propuso, mediante una revisión documental tipo metanálisis, demostrar la importancia de recopilar información científica pertinente a la nutrición alternativa en conejo a partir de Matarraton (Gliricidia sepium), Botón de oro (Tithonia diversifolia) y Morera (Morus alba). Analizando comparativamente los tres forrajes tropicales utilizados en la nutrición alternativa de conejos sobre la eficiencia productiva.

La nutrición es uno de los aspectos más importantes para la producción animal en cualquiera de las especies domésticas utilizadas para consumo humano, es así como En la cría de conejos la alimentación con concentrado representa entre el 60 y 70\% del costo total de la producción según (Miranda, et al en 2012), por lo anterior se hace necesario la búsqueda de Sistemas de alimentación alternativos de bajo costo.

Se trata pues, de buscar alternativas de alimentación con productos forrajeros del medio; que puedan facilitar la producción cunícola a menor costo sin perder la calidad y propiedades de su carne, para ello, se han realizado estudios con especies forrajeras comunes y de producción de follaje que sirven como dieta alternativa total o parcial como son las especies Botón de oro, Matarratón, y Morera, que han sido objeto de estudios en países como Cuba, Colombia, Costa Rica y Venezuela desde diferentes perspectivas de la necesidad en la dieta ya sea para la producción de carne, desarrollo en una etapa de crecimiento, cría de reproductoras como alternativa para reducción en los costos de producción.

La problemática anteriormente señalada, determina la realización de la siguiente pregunta ¿Cuáles de las materias primas alternativas utilizadas en el trópico para la alimentación Cunícola, son las más eficientes?

La importancia de este estudio radica en que el meta análisis, no necesita experimentación que es altamente costosa, en cambio, permite a partir de resultados de otras investigaciones concluir cuál es la materia prima alternativa de países tropicales más eficiente en alimentación cunícola, permitiendo decidir si la alimentación con concentrados comerciales puede sustituirse sin disminuir los indicadores de la producción cunícola,

DOI: https://doi.org/10.22490/ECAPMA.2779 
recomendando las materias primas alternativas más eficientes que permitan mejorar la eficiencia productiva. Lo anterior se realiza dado que el costo de los alimentos concentrados comerciales ha generado la búsqueda de estrategias basadas en el uso de materias primas no convencionales, que permitan obtener una mayor rentabilidad en la cunicultura. En Colombia, se cuenta con una gran variedad de fuentes alimenticias con alto valor nutricional que no han sido aprovechadas adecuadamente, de las cuales podemos resaltar el Matarratón (Gliricidia sepium), botón de oro (Tithonia diversifolia) y morera (Morus alba). La alta disponibilidad de plantas probadas o potencialmente útiles para conejos, sustenta la posibilidad de incluirlas en mezclas dietéticas balanceadas preparadas en la granja, para aprovechar la capacidad herbívora de la especie.

El conejo (Oryctolagus cuniculus) por su capacidad productiva, ciclos cortos de gestación, producción a corto plazo, alta resistencia y adaptabilidad, y cuya alimentación se basa en forrajes de muchas variedades; es uno de los animales llamados a suplir este recurso proteico tan valioso e insustituible para la alimentación humana. Los forrajes alternativos pueden ser suministradas a los animales en una forma que les permita ejecutar su comportamiento de alimentación natural, sus necesidades digestivas y debe ofrecerse balanceadamente, de acuerdo con las necesidades nutricionales (De Blas 1984, McNitt et al. 1996, Carabaño y Piquer 1998,De Blas 2009) esta posibilidad nutricional en la producción de conejo, mediante el suministro de forrajes alternativos rico en proteínas de alto valor, bajo en calorías y grasas y sumamente nutritivo de conversión y ganancia de peso similar al alimento tradicional permite obtener carme con la calidad y las propiedades óptimas para el mercado y el consumo .

Como una alternativa productiva que busca una alimentación balanceada para las explotaciones cunículas, están las mezclas de especies forrajeras y los productos de su conservación los cuales pueden ser una alternativa, en ganancia de peso, siendo un recursos nutricional económico, fácil de utilizar, natural y muy palatable para la especie, "Esta fase ha estimulado la exploración de nuevas materias primas alimenticias, con la finalidad de generar patrones de producción ajustados a la realidad social y económica del entorno en que se encuentran. Este tipo de implementación nutricional propenderá por una producción canícula cada vez más inocua segura, limpia y al alcance de las comunidades rurales de Colombia.

\section{Metodología de Estudio}

El estudio "Matarratón (Gliricidia sepium), Botón de oro (Tithonia diversifolia) y Morera (Morus alba) tres especies forrajeras como alternativa en la alimentación de conejos" es una monografía de tipo meta análisis, elaborada mediante la metodología PRISMA (Urrutia y Bonilla 2010).

El metanálisis es definido como una revisión sistemática en el cual se combinan los resultados de varios estudios que examinan la misma pregunta. Es una disciplina que revisa la literatura críticamente y combina estadísticamente los resultados de estudios previos. En esencia el metanálisis busca realizar un análisis estadístico de una colección amplia de resultados de estudios individuales con el propósito de integrar sus hallazgos.

La Metodología PRISMA o Presentación de Revisiones Sistémicas y Meta análisis. se compone de una lista de verificación de elementos y un diagrama de flujo de cuatro fases.

Fase 1. Que es de identificación, Se realiza una Identificación de las citas bibliográficas de investigaciones.

En la Fase 2. Se realiza un Cribado o eliminación de algunas de las citas bibliográficas

En Fase 3. Se realiza una Elegibilidad. Es decir, se anotan las Investigaciones elegidas 
Fase 4. Se realiza una Inclusión de las Investigaciones incluidas en la síntesis cualitativa.

Protocolo, registro y criterios de elegibilidad.

El estudio parte de una población desconocida representada por investigaciones y publicaciones especializadas teniendo en cuenta los siguientes criterios de elegibilidad:

- Publicaciones indexadas en idioma español.

- Conejos de levante, cuya alimentación estuviera basada en forrajes alternativos (morera, botón de oro y Matarratón)

- Análisis de los parámetros (consumo, ganancia diaria de eso y conversión alimenticia).

- Evaluación de la eficiencia productiva mediante análisis estadístico.

Como se trata de una población de investigaciones y publicación desconocida se utilizó el 95\% de confiabilidad y un $10 \%$ de margen de error, para calcular el tamaño dela muestra se utilizó la siguiente formula:

$$
\mathrm{n}=(\mathrm{Z})^{\wedge} 2 \times \mathrm{p}^{*} \mathrm{q} /(\mathrm{e})^{\wedge} 2 \quad \longrightarrow \mathrm{n}=(1,96)^{\wedge} 2 *(0,5)(0,5) /(0,10)^{\wedge} 2=96
$$

$Z=$ nivel de confianza $p=$ probabilidad de éxito $q=$ probabilidad de fracaso e= precisión o erro máximo admisible $\mathrm{n}=\mathrm{es}$ la muestra representativa.

Como criterios de exclusión para el análisis descriptivo se tuvo en cuenta: reseña de investigaciones. Literatura gris.

La recolección de información y de artículos científicos indexados en los buscadores de google académico utilizando las palabras claves "nutrición de conejos con forrajes alternativos", "alimentación de conejos" consumo de alimento en conejos, ganancia diaria de peso, conversión de alimentos.

Conservando el tamaño de la muestra y los factores de exclusión definidos, se revisaron un total de 96 artículos científicos relacionados con consumo, ganancia y conversión alimenticia de conejos en levante, a quienes se les suministró en la ración materias primas alternativas, y, de acuerdo a los parámetros de selección iniciales se tuvieron en cuenta finalmente 30 artículos que cumplían con cada uno de los criterios, quedando la muestra finalmente con un $95 \%$ de confiabilidad y un 10\% de margen de error para desarrollar el análisis descriptivo. El $41 \%$ del total de artículos revisados no contaron con los criterios de inclusión seleccionados para ser analizados, pues aunque son válidos o confiables como información científica, no realizaban el análisis de todos los parámetros productivos establecidos para el presente meta análisis (consumo, ganancia de peso y conversión alimenticia) evaluando en algunos casos consumo y digestibilidad, en otros estudios analizaban dos etapas de producción en cerdos (levante y ceba) sin diferenciarlas o simplemente era una reseña de tesis. En la figura 1 se pueden apreciar los pasos del proceso de selección de las investigaciones encontradas. Los datos se tabularon en hoja electrónica Excel. 


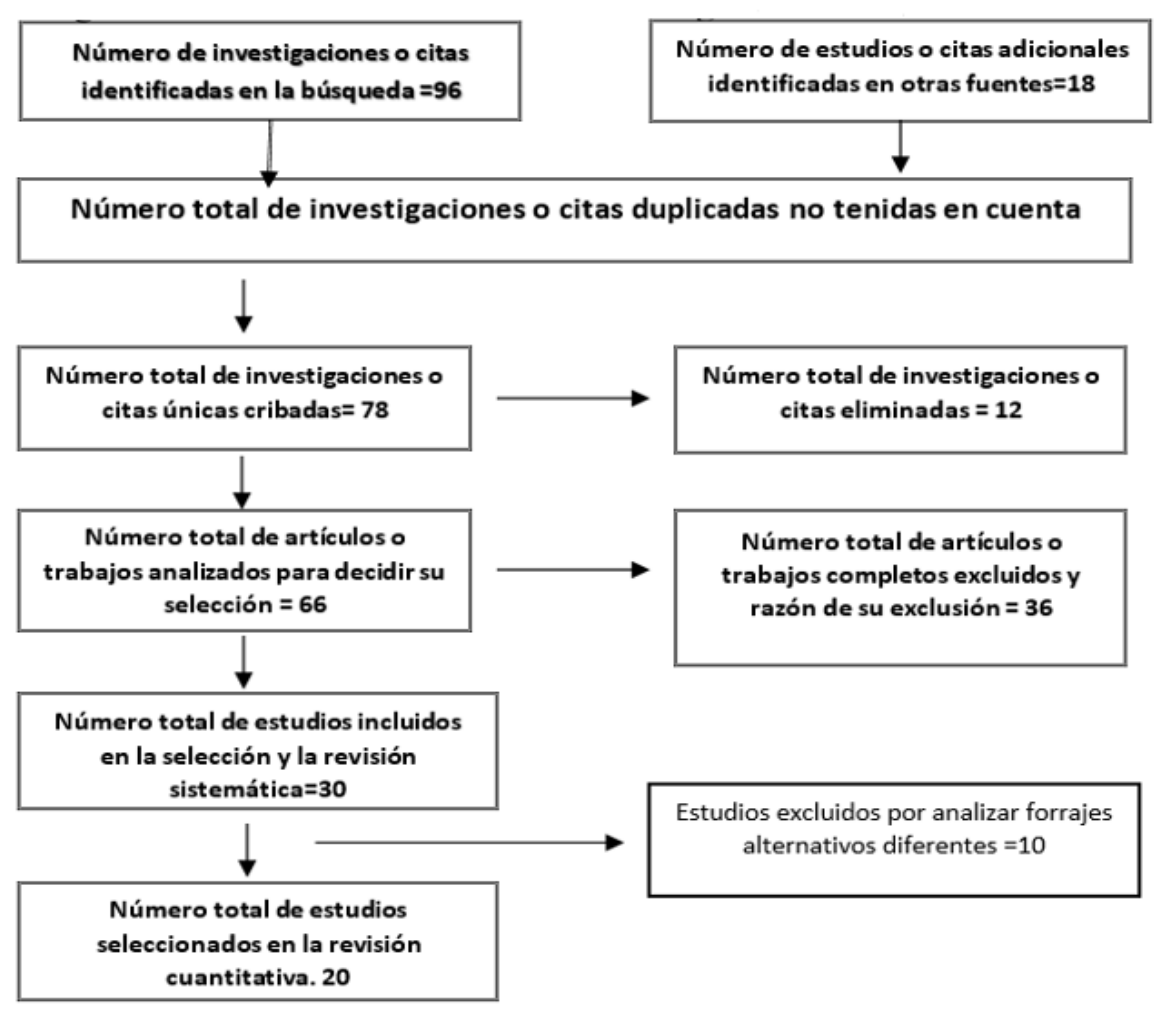

Figura 1: Proceso de selección de las investigaciones encontradas.

Fuente: Autores

Los trabajos analizados se ejecutaron en (4) países, a saber: en Cuba, Costa rica, Colombia y Venezuela. La información consultada fue de tipo secundaria, es decir, se trabajó directamente sobre bases de datos automatizadas, y no se incluyó "literatura gris" (información no publicada).

La información fue organizada, según el forraje alternativo, el país de origen de la investigación y el autor(es) de la investigación, mediante graficas de barras y análisis estadístico en Excel.

En los objetivos de tipo cuantitativo se analizaron 40 investigaciones, permitiendo determinar los promedios de cada una de las variables (consumo, ganancia de peso y conversión de alimento) para establecer diferencias significativas teniendo en cuenta tres forrajes alternativos; morera (Morus alba), Matarratón (Gliricidia sepium), Botón de oro (Tithonia diversifolia)

\section{Resultados preliminares y Discusión}

En la tabla (1) se observa el promedio de consumo por cada tratamiento, el tratamiento 1 Botón de oro presenta el mayor consumo (150gr/día) mientras el Matarraton es el menor promedio con (132 gr/día) siendo este el menor coeficiente de variación.se puede observar que ninguno supero el coeficiente de variación del $30 \%$. Esto significa que si se repite el experimento los resultados pueden ser los mismos con respecto al tratamiento de control los tres tipos de forrajes alternativos como dieta alimentaria ninguno estuvo por debajo del promedio de consumo dos tuvieron un comportamiento muy similar: Matarraton y morera." Fomunyam \& Ndoping (2000) y Nieves et al. (2009a) indican que los niveles, recomendados de PC en la dieta de conejos de engorde son 
200 y $227 \mathrm{~g} / \mathrm{kg}$ de MS, respectivamente." Se puede observar que el consumo de alimento utilizado en los experimentos fue inferior.

Tabla No. 1. Análisis estadísticos del consumo en dietas de forrajes alternativos Vs Alimento concentrado.

\begin{tabular}{lllllllll}
\hline \multicolumn{7}{c}{ consumo de forrajes alternativos vs alimento concentrado } \\
\hline tratamiento & $\begin{array}{l}\text { desviación } \\
\text { estándar }\end{array}$ & $\begin{array}{l}\text { Coeficiente. } \\
\text { de variación }\end{array}$ & mínimo & máximo & rango & $\begin{array}{l}\text { coef.de } \\
\text { simetría }\end{array}$ & Curtosis \\
$\begin{array}{l}\text { botón de } \\
\text { oro }\end{array}$ & 32,57864331 & 29,7141949 & 82,6 & 150 & 67,4 & 0,632481179 & $-2,984058$ \\
Matarraton & 11,53256259 & 9,53104347 & 109 & 132 & 23 & - & $-0,3021326$ \\
morera & 13,92875162 & 12,7067124 & 83 & 135 & 52 & 0,387265656 & - & 0,81561029 \\
Control & 14,48788264 & 14,218994 & 81,4 & 130 & 48,6 & 0,156017495 & - \\
\hline Total & 18,13119600 & 16,5427362 & 81,4 & 150 & 47,75 & 0,623847927 & 0,54060932 \\
\hline
\end{tabular}

Fuente: Autores

En la tabla (2) muestra el promedio de ganancia de peso diario por cada tratamiento en el tratamiento con Matarraton se obtuvo la menor ganancia de peso diario $(19,2 \mathrm{~g} / \mathrm{día})$. entre todos los tratamientos el aplicado con morera es el de mayor promedio de ganancia día de peso $(27,5)$ este podría ser un sustituto ideal para la cría de conejos con forrajes alternativos, puesto que en la productividad y la eficiencia se busca el menor consumo de alimento y mayor ganancia de peso." Las ganancias diarias de peso fueron similares a lo reportado por Fernández-Carmona et al. (1998) y Martínez et al. (2005), quienes reportan ganancias diarias de $37,2-40,3$ y $34-46 \mathrm{~g}$, respectivamente" muy similar a la ganancia de peso promedio hallada en el presenté trabajo.

Tabla No. 2. Análisis estadísticos de la ganancia de peso diario con dietas a base de forrajes alternativos Vs Alimento concentrado.

\begin{tabular}{ccccccccc}
\hline \multicolumn{7}{c}{ GANANCIA DE PESO PROMEDIO CON FORRAJES ALTERNATIVOS } \\
\hline Tratamiento & $\begin{array}{c}\text { desviación } \\
\text { st }\end{array}$ & $\begin{array}{c}\text { Coef. De } \\
\text { variación }\end{array}$ & mínimo & máximo & rango & $\begin{array}{c}\text { coef.de } \\
\text { simetría }\end{array}$ & Curtosis \\
botón de oro & 1,73588018 & 9,39125828 & 16 & 20,8 & 4,8 & - & 1,2194631 \\
Matarraton & 1,311487705 & 7,40953506 & 16 & 19,2 & 3,2 & 0,229232034 & - & 1,61492699 \\
morera & 4,091740017 & 19,8418005 & 14,77 & 27,5 & 12,73 & 0,356477818 & - \\
Control & 6,422080377 & 6,30288942 & 17 & 46,4 & 29,4 & 2,395595575 & 7,25044025 \\
total & 3,39029707 & 10,7363708 & 15,9425 & 28,475 & 12,53 & 2,068892127 & 2,2466694
\end{tabular}


En la tabla (3) se observa el comportamiento del promedio de la conversión alimenticia de forrajes alternativos frente al concentrado encontrándose mayores desempeños y menores desempeños entre tratamientos y frente al tratamiento de control. Se observa que el menor promedio de conversión es el tratamiento con dieta de Botón de oro; pero todos los tratamientos son de conversión inferior al tratamiento de control o sea dieta con alimento concentrado comercial.

Tabla No. 3. Análisis estadísticos de la conversión alimentaria con dietas a base de forrajes alternativos Vs Alimento concentrado.

\begin{tabular}{lccccccc}
\hline \multicolumn{7}{c}{ CONVERSIÓN ALIMENTARIA PROMEDIO CON FORRAJES ALTERNATIVOS } \\
\hline Tratamiento & $\begin{array}{c}\text { desviación } \\
\text { st }\end{array}$ & $\begin{array}{c}\text { Coef. De } \\
\text { variación }\end{array}$ & mínimo & máximo & rango & $\begin{array}{c}\text { coef.de } \\
\text { simetría }\end{array}$ & curtosis \\
botón de oro & 2,114076631 & 35,1876936 & 4,3 & 9,4 & 5,1 & 1,363144763 & 1,10125438 \\
Matarraton & 0,989949494 & 16,4991582 & 4,6 & 6,9 & 2,3 & - & 1,17234562 \\
& & & & & & 1,339998273 & \\
morera & 1,087101233 & 19,7982013 & 3,8 & 7,7 & 3,9 & 0,480571891 & 0,41060259 \\
Control & 1,014704462 & 23,4971174 & 2,76 & 6,5 & 3,74 & 0,520487709 & 0,2034469 \\
total & 1,301457955 & 23,7455426 & 3,865 & 7,625 & 3,76 & 0,256051522 & 0,72191237 \\
\hline
\end{tabular}

Fuente: Autores

\section{Conclusiones}

Los tratamientos con forrajes alternativos tropicales como la Morera (Morus alba) y Botón de oro (Tithonia diversifolia) utilizados en las dietas para los conejos neozelandeses por los diferentes autores y en los diferentes países fueron superiores al producto testigo o alimento concentro. Solo el forraje con Matarraton (Gliricidia sepium), fue suministrado en cantidad similar al alimento concentrado. Al tener en cuenta la variable conversión de alimento, en todos los casos fue mejor el comportamiento del alimento concentrado; pero la morera (Morus alba) y Matarraton ((Gliricidia sepium), en su orden tienen un buen desempeño si lo comparamos con el botón de oro (Tithonia diversifolia) que presento menor grado de conversión dentro de los forrajes que se tuvieron en cuenta para el análisis en el presente estudio.

La variable ganancia diaria de peso, mediante el suministro de alimento concentrado (Tratamiento testigo), fue superior frente a la dieta a base de forrajes alternativos tropicales morera (Morus alba), botón de oro (Tithonia diversifolia) y Matarraton (Gliricidia sepium); siendo la dieta con a base de morera (Morus alba), el forraje alternativo que presento el mejor comportamiento.

Las dietas a base de forraje alternativo botón de oro (Tithonia diversifolia) presenta el menor desempeño para la variable ganancia de peso diario y los autores no recomiendan establecerlo como dieta única, estos pueden ser una alternativa en asocio con otros forrajes como morera o

Ramio; por su aporte excepcional de nitrógeno Villa-Ramírez- Hurtado-Villegas et al 2016.

Las dietas únicas a base de forrajes Botón de oro (Tithonia diversifolia), Matarraton (Gliricidia sepium), y Morera (Morus alba) pueden poner en peligro a reproducción; se requieren estudios más profundos; puesto que en los diferentes estudios estas dietas sustituyen el alimento concentrado hasta en un $30 \%$ de la dieta total. 


\section{Literatura citada}

Alvarado Hipólito, Brea Patricia., Nouel Gustavo., Espejo Miguel, Mejía Gezminer., Sánchez Roseliano, Hevia Patricio, Romero Yosmar, (2003). Consumo y digestibilidad de bloques nutricionales para conejos, compuesto por tres forrajeras del semiárido comparadas con soya perenne. Bioagro, 15(1), 23-30. Recuperado de: http://www.ucla.edu.ve/bioagro/Rev15(1)/3.\%20Consumo\%20y\%20digestibilidad.pdf

Álvarez Ramírez, F., Castaño Arcila, G., Montes Moreno, J., \& Valencia Trujillo, F. (2013). Las escuelas campesinas de agroecología, centros de formación campesina y los custodios de semillas en los Andes tulueños (Colombia). Revista de Investigación Agraria y Ambiental, 4(2), 135-147. doi:https://doi.org/10.22490/21456453.988

Brenes. A. (2014). Respuesta productiva de conejos alimentados con follaje fresco de nacedero (Trichanthera gigantea, Lamiales: Acanthaceae). Centro de Investigaciones en Nutrición Animal y Escuela de Zootecnia, Universidad de Costa Rica. San José, Costa Rica. P. 1-8. Recuperado de: http://investiga.uned.ac.cr/revistas/index.php/cuadernos/article/view/624

Cardozo. J. (2013). El Matarraton (Gliricidia sepium) en la Alimentación de Rumiantes. (Monografía) Universidad Nacional Abierta y a Distancia - UNAD. Bogotá. Colombia. P. 66 Recuperado de: https://repository.unad.edu.co/handle/10596/1076

Castaño. G., Cardona. J. (2015). Engorde de Conejos Alimentados con Tithonia diversifolia, Trichanthera gigantea y Arachis pintoi. Santa Rosa de Cabal, Risaralda, Colombia. P. 8. Recuperado de: https://revistas.udca.edu.co/index.php/ruadc/article/view/463

Clara Inés Ríos Katto y Amparo Salazar. (1995). Botón de oro (Tithonia diversifolia (Hemsl.), Gray una fuente proteica alternativa para el trópico. Recuperado de: http://www.fao.org/AG/AGa/AGAP/FRG/FEEDback//rrd//rrd6/3/9.htm

David Mora Valverde. (2012). Evaluación de cuatro niveles de morera (Morus alba) en engorde, de conejo bajo normativa orgánica. Recuperado de: http://www.scielo.sa.cr/pdf/am/v23n2/a10v23n2

Díaz, R. G., \& Valencia, F. L. (2010). Evaluación de la sustentabilidad ambiental de tres sistemas de producción agropecuarios, en el corregimiento Bolo San Isidro, Palmira (Valle del Cauca). Revista de Investigación Agraria y Ambiental, volumen1 número (2), 7-17. Recuperado de: http://hemeroteca.unad.edu.co/index.php/riaa/article/view/900

Duilio Nieves, Omar Terán, Gloria Arciniegas, Carlos González, Julio ly. (2009). Comportamiento productivo de conejos alimentados, con dietas basadas en follajes tropicales. Recuperado de http://www.scielo.org.ve/scielo.php?pid=S0798-22592009000200011\&script=sci arttext\&tlng=en

González. J., Hahn von. Ch., Narváez. W. (2014). Características botánicas de Tithonia diversifolia (asterales: asteraceae) y su uso en la alimentación animal. Cient. Mus. Hist. Nat, Vol. 18( No 2), 45-58. Recuperado de: http://200.21.104.25/boletincientifico/downloads/Boletin(18)2 4.pdf

Henao. J., Gutiérrez. N., Oviedo. O. (2012). Uso de subproductos agrícolas en la alimentación de conejos en fases de ceba y reproducción. Revista Biotecnología en el Sector Agropecuario y Agroindustrial. Unicauca. Vol. 10 No. 2. Colombia. P. 1-7 Recuperado de: http://revistabiotecnologia.unicauca.edu.co/revista/index.php/biotecnologia/article/view/232

Hurtato. D., Nocua. S., Narváez. W., Vargas. J. (2012). Valor nutricional de la morera (Morus sp.), Matarratón (Gliricidia sepium), pasto indio (Panicum máximum) y arboloco (Montanoa quadrangularis) en la alimentación de cuyes (Cavia porcellus). Revista de Veterinária e Zootecnia, 6(1), 56-65. Recuperado de: http://vip.ucaldas.edu.co/vetzootec/downloads/v6n1a06.pdf 
Lara, P., Itzá, M., Sanginés, J., y Magaña, M. (2012). Morus alba o Hibiscus rosa sinensis como sustituto parcial de soya en dietas integrales para conejos. Universidad Autónoma de Ciudad Juárez. P. 12. Recuperado de http://www.redalyc.org/html/837/83724458002/

López. O., Montejo. I., y lamela. L. (2012). Evaluación del potencial nutricional de cuatro plantas forrajeras para la alimentación de reproductoras cunículas. Revista Pastos y Forrajes. Nota técnica. Vol. 35. No. 3. P. 1-8.Recuperado de: http://scielo.sld.cu/pdf/pyf/v35n3/pyf05312.pdf

Malavé. A., Córdova. L., García. A. Méndez. J. (2013). Composición bromatológica de la carne de conejos suplementados con Matarratón y cachaza de palma aceitera. Revista MVZ Córdoba. Vol. 18(2). Universidad de Oriente. $\quad$ P. $1-7$ Recuperado de: https://dialnet.unirioja.es/servlet/articulo?codigo $=4351427$

Mendoza Velásquez, S., Cano Muñoz, J., \& Rojas Sánchez, F. (2015). Acción comunitaria frente al fenómeno del cambio climático, en el páramo de la región del Guavio, Cundinamarca, Colombia. Revista de Investigación Agraria y Ambiental, 6(1), 265 - 279. doi:https://doi.org/10.22490/21456453.1286

Miranda. L., Rodríguez. R., González. M., \& Campos. R. (s.f.). (2012). Alimentación del Conejo con Bloques Multinutricionales y Productos de la Fermentación Microbiana. Universidad Autónoma Chapingo.

P. 6. Recuperado de: http://bit.ly/2uzD3a5.

Mora. D. (2010). Usos de la morera (Morus alba) en la alimentación del conejo. El rol de la fibra y la proteína en el Tracto digestivo. Universidad de Costa Rica. Revista Agronomía mesoamericana. Vol. 21(2). P. 1-10 Recuperado de: http://www.scielo.sa.cr/pdf/am/v21n2/a17v21n2.pdf

Mora. D. (2012). Evaluación de Cuatro Niveles de Morera (Morus alba) En Engorde de Conejo Bajo Normativa Orgánica. Inclusión de morera en producción alternativa de conejos. Revista Agronomía Mesoamericana 23(2). Universidad de Costa Rica. P. 9. Recuperado de: http://www.scielo.sa.cr/pdf/am/v23n2/a10v23n2

Morh J (2000) Mejora de la calidad de los metanálisis de ensayos aleatorios. Recuperado de: https://es.cochrane.org/sites/es.cochrane.org/files/public/uploads/PRISMA Spanish.pdf

Nieves. D. (s.f.). Forrajes Promisorios Para la Alimentación de Conejos en Venezuela. Valor Nutricional. Alimentación no convencional para monogástricos en el trópico. Universidad Ezequiel Zamora, Unellez, Guanare. $\quad$ P. 14 Recuperado de: http://avpa.ula.ve/eventos/viii encuentro monogastricos/curso alimentacion no convencional/confe rencia-2.pdf

Nieves. D., Pérez. J., Jiménez. N., Calles. H., Pineda. T., y Viloria. W. (2012). Uso de follaje fresco de árnica (Tithonia diversifolia) y morera (Morus alba) en la alimentación de conejos. Revista Academia, Vol. 11 No (22), 113-123. Recuperado de: https://dialnet.unirioja.es/servlet/articulo?codigo=5166283

Nieves. D., Rojas. E., Terán. O., Fuenmayor. A., \& González. C. (2005). Aceptabilidad de dietas con naranjillo, leucaena, morera, maní, forrajero. Batata, y yuca en dietas para conejos de engorde. Revista Unellez Ciencia tecnológica. Vol. 23. P. 7. Recuperado de http://revistas.unellez.edu.ve/revista/index.php/ruct/article/view/28/25

Nieves. D., Terán. O., Vivas. M., Arciniegas. G., González. C., y Ly. J. (2009). Comportamiento Productivo de Conejos Alimentados con Dietas Basadas en Follajes Tropicales. Revista Científica de veterinaria, Vol. 19, № 2. P. 9. Recuperado de: http://www.scielo.org.ve/scielo.php?pid=S0798$\underline{22592009000200011 \& \text { script=sci arttext\&tlng=en }}$

Nouel. G., Espejo. M., Sánchez. R., Hevia. P., Alvarado. H., Brea. A., Romero. Y., y Mejías. G. (2003). Consumo y digestibilidad de bloques nutricionales para conejos, compuestos por tres forrajeras del semiárido comparadas con soya perenne. Bioagro. Vol. 15 (1). P. 1-8 Recuperado de: http://www.scielo.org.ve/scielo.php?pid=S1316-33612003000100003\&script=sci arttext\&tlng=en 
Pérez. A., Montejo. I., Iglesias. J., López. O., Martín. G. García. D., Idolkis. M. Hernández. A. (2009). Tithonia diversifolia (Hemsl.) A. Gray. Pastos y Forrajes. Instituto Nacional de Investigaciones Agrícolas, Estado Trujillo, Venezuela. Vol. 32, No. 1. P. 15. Recuperado de: http://scielo.sld.cu/scielo.php?script=sci_arttext\&pid=S0864-03942009000100001

Quintero P., Victoria E.; García R., Genny P.; Peláez R., Angélica M. (2007). Evaluación de harina de botón de oro en dietas para conejos en etapa de crecimiento. Revista Acta Agronómica, vol. 56, núm. 4, pp. 203-206 Universidad Nacional de Colombia. Palmira, Colombia Recuperado de: http://www.redalyc.org/pdf/1699/169913316008.pdf

Ríos. C. (s.f.). Tithonia diversifolia (hemsl.) Gray, una planta con potencial para la producción sostenible en el trópico. Agroforestería para la Producción Animal en Latinoamérica. Fundación Centro para la Investigación en Sistemas Sostenibles de Producción Agropecuaria. Cali, Colombia. P. 217-230. Recuperado de: http://www.fao.org/ag/aGa/agap/FRG/AGROFOR1/Rios14.PDF

Sánchez. A., Torres. E., Meza. G., Estupiñán. K., Torres. Y., Barrera A., Mackencie. Y., ～López. ～L., (2012). Efecto de dos leguminosas y banano maduro en la producción y reproducción de conejos Nueva Zelanda. Artículo científico en Ciencia y Tecnología. Vol. 5 No (2). P. 1-5 Recuperado de: http://autoevaluacion.uteq.edu.ec/index.php/cyt/article/view/84

Urrutia y Bonilla (2010). Declaración PRISMA: una propuesta para mejorar la publicación de revisiones sistemáticas y metanálisis. Recuperado de: https://es.cochrane.org/sites/es.cochrane.org/files/public/uploads/PRISMA Spanish.pdf

Valdivié, M., \& Ponce de León, R. (2015). Las investigaciones cunícolas en Instituto de Ciencia Animal. Revista Cubana de Ciencia Agrícola, 49(2). Recuperado de http://www.redalyc.org/html/1930/193039698010/

Vásquez. Y., Valdivié. M., Berrios. I., \& Sosa. E. (2016). Sistemas de alimentación con forraje de Morus alba y tallo de caña de azúcar para conejos en ceba. Revista electrónica de Veterinaria. Volumen 17 № 12. Instituto de Ciencia Animal. San José de las Lajas. Mayabeque. Cuba. P. 1-7. Recuperado de: http://www.redalyc.org/html/636/63649052011/

Villa. R., \& Hurtado. J. (2016). Evaluación del peso de conejos para carne alimentados con diferentes ensilajes. Revista Ciencia y Agricultura (Rev. Cien. Agri.) Vol. 13 (2). P. 73- 81. Tunja (Boyacá) Colombia. Recuperado de: https://dialnet.unirioja.es/servlet/articulo?codigo $=5712001$ 